\title{
Accidental Nail Gun Penetration to Right Ventricle: A Case Report
}

\author{
Yasser Shabaan Mubark and Ahmed Mohamed Farghaly* \\ Department of Cardiothoracic Surgery, Minia University Hospital, Minia University, Egypt
}

Submission: February 05, 2017; Published: March 08, 2017

*Corresponding author: Ahmed Mohamed Farghaly, Department of Cardiothoracic Surgery, Minia University Hospital, Minia University, Egypt, Email: ahmedmfarghaly14@gmail.com

\begin{abstract}
Introduction: Penetrating cardiac injuries are life-threatening conditions that require urgent surgical intervention, and often associated with a high mortality rates. Work site injuries by nail-gun are present, and although usually involve the limbs, penetrating cardiac injuries can occur.

Case presentation: We report a case of a 21 years old carpenter who accidentally shot himself with a nail gun. An emergent chest computed tomography was done and revealed a nail penetrating the right ventricle with massive haemopericardium. Median sternotomy was performed, followed by nail removal and repair of the right ventricular wound. He had an uncomplicated postoperative course and was discharged 7 days postoperatively.
\end{abstract}

Conclusion: Cardiac nail gun injuries are rare conditions but fatal. They require emergent convenient surgery, and timing of intervention should be considered to obtain best outcomes.

Keywords: Chest trauma; Nail gun; Penetrating injury; Cardiac tamponade; Heart ventricles

\section{Introduction}

The incidence of accidental injuries of nail guns is increasing, as these devices can be obtained easily and in turn used with minimal experience and training [1]. Although, most of these injuries usually involve the limbs [2], a small proportion of penetrating cardiac injuries have been reported with high morbidity and mortality rates [3]. Almost all such injuries are in adult men [1]. Moreover, these penetrating injuries can develop cardiac tamponade particularly during time-consuming examinations as computed tomography (CT) that leads to hemodynamic instability and more aggravation. Accordingly, those patients should be transferred rapidly to the operating room for emergent surgery [4]. The treatment provided to these injuries is either median sternotomy or antero-lateral thoracotomy, followed by decompression of the pericardium and repair of the cardiac injury [5]

\section{Case Presentation}

During his work in January 2017, a 21 years old carpenter overbalanced and shot himself with a nail gun. The injury was in the right hemithorax at the level of $4^{\text {th }}$ intercostal space $1 \mathrm{~cm}$ lateral to the right of midline, and appeared as a small, lightly bleeding wound.
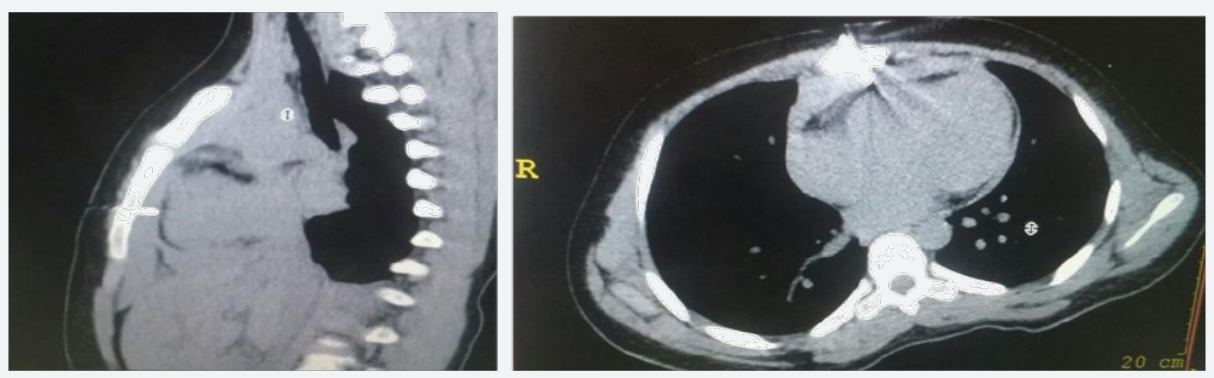

Figure 1: Chest CT showing a nail penetrating the right ventricle. 
The patient was delivered to our emergency center collapsed, as he was hypotensive (51/39), tachycardic (163) with prominent jugular veins. Immediate resuscitation (central venous access, perfusion with blood products) was done by the anaesthesia team that stabilizes the condition and enabled CT scan, which showed a penetrating cardiac injury with retained fired nail and massive haemopericardium Figure 1. Sudden circulatory collapse occurred presented with hypotension, progressive bradycardia, and congested neck veins, so the patient was taken immediately to the operating room.

Median sternotomy performed, followed by pericardiotomy to evacuate massive amount of blood and clots from the pericardial sac. Haemodynamics improved as soon as we opened the tense pericardium, and circulatory failure was overcome, but sinus tachycardia began. Inspection of the surgical field revealed a nail penetrating the anterior surface of the right ventricle. The nail removed simply and the myocardium repaired with 3-0 pledgetted Prolene transverse mattress sutures without using cardiopulmonary bypass. Intraoperative transesophageal echocardiography confirms no valvular or septal injury. A chest tube (32 French) was left retrosternal followed by sternal closure.

Postoperatively, the patient was given antibiotics for 6 days to prevent potential infection from foreign material, and after smooth recovery course, the patient was discharged home on the $7^{\text {th }}$ postoperative day.

\section{Discussion}

Nail guns are one of the most commonly used devices in the construction industry, as they have the power to force a nail into wood, brick, or even concrete surfaces rapidly and easy. Although their injuries are predominantly involving the extremities, few cases of cardiac injury were reported, and they are potentially fatal carrying about $25 \%$ risk of death [1]. This, may owing to failure of primary diagnosis, as these injuries usually presented as small penetrating wound with unrecognizable foreign body, besides no classical signs of tamponade that lead to an underestimation of the cases [6], and that was in consisting with our experience.

As in other penetrating cardiac injuries, pericardial tamponade may develop; at first it is life-saving, but turns over time to be fatal, due to cardiac failure, especially when decompensation no longer responds to volume supply. In these emergent setting, bedside sonographic assessment is valuable for evaluation of contractility and pericardial effusion [7]. On the other hand, if patient is hemodynamically stable, CT with continuous monitoring should be performed, as done with our patient to accurately detect the site of the nail and the extent of injury [2].

Treatment of nail gun injuries mostly requires emergency operation. Either median sternotomy or anterolateral thoracotomy can be performed depending on the site of injury, aiming to relief the tamponade and surgical repair of the wound. Besides to surgery, fluid resuscitation is critical [7]. In rare cases, cardio pulmonary bypass may be needed, if other cardiac lesions, as; septal defects or heart-valve injuries are present [2].

\section{Conclusion}

In conclusion, although cardiac nail gun injury is rare, it can occur and are survivable. This case demonstrates that physical examination has a significant role in prompt diagnosis, with special attention to chest wall examination. Moreover, CT scanning is mandatory to identify the nail site exactly, extent of injury, and the presence of haemopericardium that may develop tamponade. Our experience with such these cases reveals that right ventricle is commonly involved due to its anterior position, and the key to survival is the accurate recognition of the nature of the injury, and the timing of surgical intervention.

\section{Consent}

Written informed consent was obtained from the patient for publication of this case report and accompanying images.

\section{References}

1. Temple AD, Fesmire FM, Seaberg DC, Severance HW (2013) Cardiac injury due to accidental discharge of nail gun. J Emerg Med 44(2): e161-e163.

2. Comoglio C, Sansone F, Boffini M, Ribezzo M, Rinaldi M (2010) Nail gun penetrating injury of the heart mimicking an acute coronary syndrome. Int J Emerg Med 3(2): 135-137.

3. Guo LR, Myers M (2008) Penetrating cardiac injury: the nail gun, a potentially dangerous tool. Can J Surg 51(1): E7-E8.

4. Hsia RY, Mahadevan SV, Brundage SI (2012) Penetrating cardiac injury from a wooden knitting needle. J Emerg Med 43(1): 116-119.

5. Chirumamilla V, Prabhakaran K, Patrizio P, Savino JA, Marini CP, et al. (2016) Pericardiocentesis followed by thoracotomy and repair of penetrating cardiac injury caused by nail gun injury to the heart. Int J Surg Case Rep 23: 98-100.

6. Nölke L, Naughton P, Shaw C, Hurley J, Wood AE (2005) Accidental nail gun injuries to the heart: diagnostic, treatment, and epidemiological considerations. J Trauma 58(1): 172-174.

7. Rupprecht $H$, Ghidau M (2014) Penetrating nail-gun injury of the heart managed by adenosine-induced asystole in the absence of a heart-lung machine. Tex Heart Inst J 41(4): 429-432. 


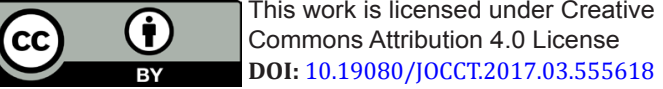

